\title{
EL ARTE
}

EN LA EDUCACIÓN,

EN BÚSQUEDA DE UN

NUEVO EPISTEMA

EDUCATIVO

Andrés Hermann Acosta

Docente Universidad Politécnica Salesiana

El arte, por tanto, es uno de los instrumentos más poderosos que disponemos para la realización de la vida, negar esta posibilidad a los seres humanos es ciertamente desheredarlos.

Rudolf Arheim ${ }^{1}$

Entre los aportes significativos que brinda la filosofía en el campo del arte está la posibilidad de cuestionar los diversos problemas en torno a la construcción del conocimiento. De esta manera surge la necesidad de interpretar al arte como la construcción de un nuevo epistema educativo, el cual denominaremos pensamiento lateral. ${ }^{2}$ Esta premisa pretende comprender el arte como una expresión que va más allá del plano estético y cultural.

En este sentido, desde una primera aproximación conceptual podemos referirnos al arte como la creación de ideas, representaciones y simbologías realizadas 
por el ser humano. Pero para que el arte pueda ser entendido como un nuevo epistema educativo deberá crearse una ruptura en torno a la visión tradicional entorno a lo estético, para ir hacia la articulación de la epistemología de la educación como una forma de posibilitar un nuevo escenario para la reflexión, construcción del conocimiento y confrontación de la realidad social.

Esta última premisa se refiere al arte a partir de expresiones que lo vinculan con la inconformidad frente al sistema, la crítica del poder, de las instituciones, la crisis del ser humano. Estos aspectos han sido abordados en muestras como las del pintor español Salvador Dalí, quien a partir de sus obras logra plasmar la descomposición de la sociedad española en la primera mitad del siglo XX generada por el régimen político dictatorial.

De tal manera, el arte en el desarrollo de la historia ha mostrado los rasgos de cada cultura. En el caso de Grecia, permitió articular la filosofía como forma de producción del pensamiento, a partir de la literatura y la mitología, que ayudaron a explicar los rasgos de la idiosincrasia de la época. La importancia de la literatura se ha examinado además desde el punto de vista que muestra las diferentes tradiciones, costumbres y construcciones del pensamiento del ser humano.

Esta premisa se ajusta a la idea del arte como un intercambio de significados, sentidos y saberes. Así, el arte ha tenido el rol de interpretar la realidad social como nuevas formas posibles en torno a la comprensión del conocimiento, no sólo desde un pensamiento vertical-lógico, sino desde un pensamiento lateral que se desarrolla a partir del pensamiento creativo, el cual no ha sido trabajado en el sistema educativo tradicional.

Mientras que en Roma el arte reflejó la opulencia del poder a partir de su arquitectura. Esto quiere decir que además de haber simbolizado la superioridad de 
algunas naciones, permitió la construcción de nuevas formas de pensamiento a través del dominio de la técnica. En esta lógica, Aristóteles refería la producción de la técnica y la razón a partir de las expresiones artísticas.

En la cultura egipcia, en cambio, constituyó una forma de mostrar la vida como preparación para la muerte. Así se vinculaba al misticismo como una forma de representar los aspectos más importantes del pensamiento de esta cultura.

En la Edad Media, se observa que la cultura se había vinculado con la filosofía escolástica y los temas religiosos propugnados por el sistema dominante del dogma. No hay que olvidar que en este momento histórico el arte pictórico, posibilitó una forma de educar al pueblo, que no sabía leer ni escribir, además de presentarse como un poderoso instrumento para la evangelización. En esta perspectiva, el arte aportó a la religión a partir de hechos como los de la persecución al cristianismo, donde disfrazaba sus símbolos, desde diversas formas como la cruz incrustada en círculos (crux dissimulata). Esto permitió ocultar los símbolos de la religión, sin exponerlos a la oposición del Catolicismo, logrando así mantener viva la tradición religiosa a lo largo de la historia.

En el siglo XX el arte poseerá gran incidencia en el orden político, donde logrará hacer fuertes críticas al sistema. Así, el muralismo mexicano constituía una expresión de crítica al sistema político. Esto será de gran impacto ya que la visión del arte en este momento histórico trascendía de un plano individual-estético hacia lo colectivo y social. Tales aspectos reflejan además una forma de cuestionamiento del ser humano como parte de la crisis social y existencial.

Desde este punto de vista habrá que destacar la importancia del arte como una manera de interpretar la realidad social, articulando la comprensión epistémica 
como la búsqueda del problema del conocimiento. En esta perspectiva, Martín Heidegger se referiría al arte en la filosofía como la representación del sentido de la interpretación, es decir, que se vincula al arte con una forma de construcción dialéctica y ontológica del pensamiento a partir de la comprensión del saber y del ser.

Esta última idea representa la comprensión del arte en la filosofía como una forma de establecer la verdad y el acontecer (Heidegger); además, como la comprensión del ser humano partiendo de nuevas formas de pensamiento. El estudio de Pérez Ulloa establece el pensamiento lateral como nueva entrada del conocimiento, el mismo que tendrá que dialogar con el pensamiento lógico-vertical vinculado con el conocimiento tradicional, que se ha desarrollado desde las ciencias exactas, asignatura que ha ocupado parte central de los currículos educativos.

En esta perspectiva, el pensamiento lateral puede constituirse en un nuevo epistema educativo, caracterizado por desarrollar destrezas como la creatividad, expresividad y sensibilidad, medios de desarrollo de un pensamiento liberador (Pérez Ulloa, 2002: 82). Este tipo de pensamiento, como ya se destacó, se construye a partir del arte, posibilitando nuevas formas de interpretar y conocer la realidad a través del campo de los sentidos y la praxis.

En esta lógica, el pensamiento lateral deberá apoyarse en otras estrategias para re-semantizar el epistema educativo, tomado en cuenta que el problema del pensamiento vertical o tradicional en la educación no radica en la razón (logos) como expresión del pensamiento universal y totalizante, sino que reside en la instrumentalización de la razón.

Vincular el arte a la educación, permite re-plantear el enfoque educativo. Junto al arte, será necesario unir además los saberes sociales. Se pretende entonces re- 
semantizar la instrumentalización de la razón a través del arte como estrategia que permita la construcción de un nuevo modelo educativo, que vuelva posible que el educando se acerque y apropie del conocimiento.

Desde esta perspectiva, Lander plantea la revitalización de las tradiciones, saberes, identidad y cultura, que se atribuyen también al campo del arte y la educación, como pensamiento divergente ${ }^{3}$, que deberá dialogar con el conocimiento tradicional (Lander, 1993: 15). Esto quiere decir que el pensamiento lateral posibilitará al educando nuevas formas de interpretar la realidad a través del pensamiento creativo-liberador.

Para Rudolf Arheim, la construcción de este nuevo epistema se vincula con la importancia de tomar en cuenta a la filosofía como punto de construcción entre pensamiento lógico y lateral (Arheim, 1993, 89). Todo esto con la idea de articular estas dos formas de conocimiento en el ámbito educativo, potenciando así los aprendizajes, mejorando la organización de los nuevos conceptos y contenidos, generando una visión del arte como estrategia dinamizadora en los procesos educativos.

\section{Estética: una visión desde el arte y la ilustra- ción ${ }^{4}$}

En la modernidad y en la ilustración, el arte debió responder a principios como la simetría, el equilibrio, la armonía y el movimiento de las formas, que desde la lectura del arte moderno ya no tienen vigencia. El concepto de estética se ha ido modificando, lo que se entendía como estético en una etapa histórica, en otra habría cambiado; así la estética ha dependido de acuerdos sociales como la política, la moral, la religión, entre otros aspectos. 
De esta manera surge un primer interrogante: ¿El arte deberá ajustarse a los principios de estética para ser considerado como tal? Surge una primera respuesta: el arte no necesariamente tendrá que ajustarse a los principios de la estética que se manejaron en la Ilustración, ya que el gusto ha ido cambiando en cada etapa de la historia. Así, en el periodo barroco lo que se caracterizaba como estético fue la excesiva recarga en los decorados, principio que mostró los rasgos de una sociedad europea pretenciosa y opulenta, que quiso poner en evidencia su poderío bélico, político y económico a través del arte.

De este último criterio surge un segundo interrogante: ¿El arte deberá ajustarse en un concepto de lo bello? Si tomamos en cuenta los aspectos antes mencionados se podría establecer que no todo lo que es bello es estético. Este criterio entendido desde una dimensión amplia que vincula al arte de acuerdo al momento histórico que se desarrolla. Es decir, que si el ideal del arte en el periodo clásico fue la simetría de las formas, en el contemporáneo sería la asimetría; este último concepto no necesariamente se ajusta a la idea de lo estético, sino a una forma de plasmar la decadencia del ser humano y su crisis social.

Los aspectos anotados explican cómo las nuevas tendencias del arte han ido adquiriendo nuevas formas de interpretar el sentido de lo estético, así en el caso del feísmo, el concepto de estética sería provocar efectos contrarios a los de la simetría de las formas, con la idea de mostrar lo desagradable y así despertar interés y aceptación en los espectadores.

Otro concepto de lo estético surge de Immanuel Kant. Este filósofo se refiere a aquello que causa placer y sensibilidad a partir de lo bello y lo sublime, entendido como una sensación de agrado (Kant, 1988: 3). El estudio 
de Kant destaca además que en el arte siempre estará implícito lo bello y lo sublime. El primer concepto es entendido como aquello que encanta, mientras que el segundo como aquello que conmueve. Con este último criterio podemos ubicar en ambos conceptos la idea de que el arte es aquello que conmueve y provoca sensibilidad en el ser humano.

Lo bello y lo sublime representan una expresión de lo estético que supera la apreciación de la obra de arte desde una primera percepción de los sentidos, para internarse en los estados de ánimo de la persona; es decir, que en lo bello y lo sublime confluyen las emociones; así, el terror, lo melancólico, el drama y la comedia se encuentran entrelazadas; la emoción de lo sublime es más poderosa que la emoción de lo bello (Kant, 1988: 4).

\section{El arte como expresión de comunicación y educación}

El arte además de representar una forma de pensamiento, ha constituido un elemento importante para la comunicación. Este aspecto se ha podido evidenciar en los vestigios que datan de miles de años atrás, encontrados en las cuevas de Altamira en España, en donde el ser humano plasmó en los grabados y el arte rupestre las expresiones más importantes de su paso por la historia.

En esta lógica, el arte ha desempeñado históricamente una alta función social, que ha hecho de su actividad además de un medio de comunicación y socialización, una forma de educación que, en el caso del arte pictórico, se ha podido comprender desde que el niño -antes de ir a la escuela- empieza a garabatear; incluso desde cuando aprende a dibujar antes que a escribir. 
La actividad artística en el ser humano se presenta de manera espontánea y natural desde los primeros años de vida. El niño dibuja todo lo que conoce, le agrada y siente, esto no sólo en la escuela sino también en el hogar. Es, entonces de destacar la importancia de las actividades artísticas en la escuela desde una comprensión del desarrollo de la creatividad y la inteligencia emocional; en especial del dibujo, como expresión del pensamiento lateral en el educando.

Pero la idea de que el dibujo constituye una estrategia importante en la educación es reciente, ya que en la Edad Media las artes estaban dividas en: mecánicas y liberales, estas últimas "requerían de mayor aplicación del intelecto", mientras que en el caso de las artes mecánicas (entre estas el dibujo y la pintura), serían descartadas, ya que se pensaba que no demandaban de mayor aplicación en la producción del pensamiento. Este principio además tendría sustento en el trivium y el quadrivium de la época, donde la gramática, retórica, dialéctica, aritmética, geometría, música y astronomía ocupaban parte central del currículo de la educación en el medioevo.

Este último aspecto quizá constituya una respuesta de por qué se ha dado poca importancia a las asignaturas vinculadas al campo del arte en los currículos de las instituciones educativas, exceptuando el caso de la música. Estas asignaturas más bien han sido consideradas como actividades vinculadas a la recreación del educando, dejando a un lado la importancia de la construcción del pensamiento creativo, relacionado con la inteligencia emocional, creatividad y sensibilidad, que muchas veces no ha sido abordado por el pensamiento vertical.

El arte en la educación promueve un tipo de protagonismo, ya que el educando puede interpretar un instrumento musical, pintar un cuadro, narrar una historia; estas posibilidades no son ejercidas en el caso de asig- 
naturas tradicionales. Este aspecto se ajusta de alguna manera a la visión que tuvo la Educación Liberadora a partir de uno de sus exponentes más representativos, el pedagogo brasilero Paulo Freire, quien destaca la importancia del educando como parte central del proceso de enseñanza y aprendizaje. Desde esta lógica, se podría decir que el arte posibilita al educando un tipo de centralidad en la educación, promoviendo así la construcción de individuos críticos, reflexivos y protagonistas de los nuevos cambios sociales.

Para Arheim, el arte además de promover un pensamiento creativo y un protagonismo social, ha posibilitado la comprensión de aspectos como la percepción y el desarrollo entre el pensamiento vertical y lateral. Este principio puede ser explicado tomando a la música como expresión artística, en la que se encuentra presente el pensamiento lógico desde la cadencia, el ritmo, la melodía y la armonía como expresiones del lenguaje abstracto y el pensamiento creativo a partir de la composición, ejecución e interpretación de cada uno de los instrumentos musicales.

El sistema educativo tiende a ver al arte como asignatura de relleno en los currículos educativos, ya que desde la tradición de una educación funcionalista se ha priorizado la profesionalización antes que la construcción del conocimiento. La matemática y la gramática han sido una forma de evitar que el educando piensen, y sólo reproduzca la lógica del mercado laboral. Pensamos entonces, que una de las tareas primordiales de las instituciones educativas sería fortalecer los currículos educativos con asignaturas como: música, teatro, expresión corporal, literatura, artes plásticas, con la idea de complementar y enriquecer a las otras materias que desarrollan detrezas verbales y numéricas, relacionadas con la tradición del pensamiento lógico, las que han conseguido 
dominar los planes de estudios (Arheim, 1993: 78). En este sentido, el arte constituye una estrategia importante en la educación, ya que presenta los nuevos conocimientos y contenidos de manera fácil y dinámica, generando así en el educando un mayor interés.

Así la búsqueda del nuevo epistema educativo se apoya en la pedagogía del arte como una forma de pensamiento lateral o creativo, el cual posibilita crear condiciones adecuadas para la construcción de educandos sensibles, reflexivos y críticos frente a los diversos problemas de la sociedad. Con esto no sólo estará posibilitando un pensamiento creativo, escaso en el sistema educativo tradicional, sino que estará siendo un dinamizador de los procesos de enseñanza y aprendizaje como puente entre el pensamiento lógico y las emociones; es decir, entre el cerebro y el corazón (Pérez Ulloa, 2002: 16).

En tal sentido, la respuesta de porqué la importancia del arte en la educación no sólo responde a la idea del desarrollo del pensamiento creativo, como antes se destacó, sino también del principio que ha referido Pérez Ulloa en torno al arte como una forma de adaptarse a los cambios y a solucionar los problemas de manera original, lo que permitirá al educando la habilidad de reacomodar ideas y conceptos hacia una nueva manera de hacer uso imaginativo de su pensamiento (Pérez Ulloa, 2002, 83).

Otra de las ventajas que aporta el arte, es la creación de un clima de trabajo adecuado en el aula, donde queda a un lado el clima de enseñanza y aprendizaje rígido y lleno de formalismos. Las actividades artísticas permiten que el educando aprenda en un ambiente de confianza, envuelto en un clima de magia, juego y entretenimiento. El arte como parte de una propuesta lúdica, ha de generar un ambiente óptimo, donde el educando y el docente establezcan lazos de afectividad y confianza. De 
esta manera, el educando se sentirá en un ambiente familiar, sin presiones, lo que favorecerá el desarrollo del aprendizaje (Pérez Ulloa, 2002, 87).

La importancia del arte en la educación no radica en la elaboración de la técnica artística, sino en aspectos como la comunicación, la metodología y goce del aprendizaje, logrando establecer condiciones adecuadas para que el educando pueda acercarse al conocimiento, posibilitando al niño el poder expresarse y tener una infancia feliz (Pérez Ulloa, 2002: 96).

Esta idea se ajusta a entender al arte como un escenario donde el educando se siente protagonista y centro del proceso de enseñanza y aprendizaje, además de hacer de la educación una construcción de sentidos, comunicación, pensamiento lateral e interaprendizaje en el desarrollo de un nuevo epistema educativo.

En suma, el arte en la educación deberá constituirse en un elemento de comprensión de la realidad social, una estrategia donde el pensamiento divergente constituya una forma de conocimiento creativo que haga posible que el educando solucione los problemas de manera original.

\section{Conclusión}

La posibilidad que ha brindado la filosofía en torno a la comprensión del arte en la educación es la de cuestionar la construcción del conocimiento. De esta manera ha surgido la necesidad de presentar un análisis que intente establecer una nueva forma de pensamiento denominado lateral, que ha sido impulsado por el arte, con el sentido de establecer la búsqueda de un nuevo epistema educativo que articule aspectos tales como la creatividad y la sensibilidad en el educando. 
El pensamiento artístico ha sido descuidado por parte del pensamiento lógico, que ha privilegiado una lógica que, a su vez, ha priorizado la profesionalización y el mercado laboral, antes que la construcción del aprendizaje.

En este sentido, la construcción del pensamiento apoyado en el arte toma en cuenta a este como una expresión que va más allá de un plano estético y se dirige hacia la comprensión de los distintos rasgos culturales, intercambio de significados, sentidos y saberes. De esta manera, el arte puede incidir en otros escenarios como el político, convirtiéndose en una crítica al sistema social. Esto tiene un importante impacto, trascendiendo de un plano estético-individual a un plano colectivo-social.

Como parte de esta propuesta, Edgardo Lander se plantea fortalecer el pensamiento lógico y creativo a través de nuevas miradas conceptuales que vinculen, además del arte, a los saberes sociales, revitalizando las tradiciones, la identidad y la cultura como formas de crear una ruptura ontológica entre el mundo y la razón, con el sentido de que el educando pueda acercarse y apropiarse del conocimiento.

Hemos evidenciado que no todo lo estético es bello, ya que desde el análisis del arte en la Ilustración se ha comprendido que el sentido de lo estético ha respondido a la suma de acuerdos sociales. De esta manera la propuesta que presenta el arte en el actual momento es replantear el sentido de lo estético, entendiéndolo no sólo como lo simétrico, sino también como aquello que conmueve y logra incidir en el pensamiento del ser humano.

Otro de los aportes del arte para la construcción de un nuevo epistema educativo es brindar al educando la posibilidad de que retome la centralidad en el proceso de enseñanza y el aprendizaje, promoviendo así sujetos críti- 
cos, reflexivos y actores de los cambios sociales. Es por eso que el arte en el sistema educativo no sólo deberá entenderse como una asignatura de relleno en los currículos educativos, sino desde una perspectiva que permita la construcción del pensamiento divergente mediante el desarrollo de aspectos que no toma en cuenta el pensamiento tradicional como la capacidad para adaptarse a los cambios, brindar soluciones originales y reacomodar nuevas ideas y conceptos. No se descarta aquí la importancia del pensamiento lógico, ya que el gran problema epistémico no ha sido en sí la razón, como modelo universal y totalizante, sino la instrumentalización del conocimiento.

Finalmente, el arte en la educación además de constituir un escenario para la construcción de sentidos, pensamiento creativo e interaprendizaje, deberá entenderse como un nuevo escenario donde el educando puede interpretar la realidad social, desde el desarrollo de la inteligencia emocional y de un pensamiento divergente emancipador.

\section{Notas}

1 Filósofo y psicólogo alemán; sus estudios se centraron en la Historia del Arte, Psicología del arte, Arte visual, Arte y estética; ha ejercido la cátedra universitaria en Harvard y Michigan.

2 Concepto trabajado por Iris Pérez Ulloa, que propone la construcción de un pensamiento creativo a partir del arte, en contraposición del pensamiento vertical fundamentado en el conocimiento lógico-tradicional.

3 El pensamiento divergente fue acuñado por Edward de Bono y representa una expresión de pensamiento creativo-alternativo, que ha pretendido crear una ruptura en torno al pensamiento lógico como única forma para llegar a la verdad y al conocimiento.

4 La Ilustración se ha definido como un movimiento intelectual, histórico, filosófico y artístico que alcanzó su máxima expresión en el siglo XVIII, entendida además como un periodo del Neoclasi- 
cismo, que se caracteriza por llegar al conocimiento a partir de la razón como máxima expresión de ese momento histórico. En el arte, la Ilustración comprende la estética que propugnó el equilibrio, la armonía y la simetría de las formas.

\section{Bibliografía}

ARHEIM, Rudolf

Consideraciones sobre la educación artística. Barcelona: Paidós.

HEIDEGGER, Martín

1996 El origen de la obra de arte. Barcelona, Alianza.

KANT, Immanuel

1990 Lo bello y lo sublime: ensayo de estética y moral. Madrid: Calpe.

PÉREZ ULLOA, Iris

Didáctica de la educación plástica. Buenos Aires: Magisterio del Río de la Plata.

LENDER, Edgardo

2002 Ciencias Sociales: Saberes Coloniales y Eurocéntricos. Buenos Aires: CLACSO.

Varios autores

Metodología de la educación plástica en la edad infantil, La Habana: Pueblo y educación. 